\title{
Oral nutritional supplements for preventing surgical site infections: protocol for a systematic review and meta-analysis
}

\author{
Nicholas Ralph ${ }^{1,2,3^{*}}$ (D) Lindsay Brown ${ }^{4}$, Kristy L. McKillop ${ }^{5}$, Jed Duff ${ }^{6}$, Sonya Osborne7, Victoria R. Terry ${ }^{1}$,
} Karen-Leigh Edward ${ }^{8}$, Rachel King ${ }^{9}$ and Edward Barui ${ }^{5}$

\begin{abstract}
Background: Surgical site infections (SSIs) are among the most common healthcare-associated infections. Undernutrition is an important risk factor for SSIs and can lead to delayed wound healing and longer hospital stays. Oral nutritional supplements are prescribed to reduce the risk of infection and improve health status, but data from randomised controlled trials (RCTs) have shown mixed results. Thus, the objective of our planned systematic review is to evaluate oral nutritional supplements on preventing SSIs in adult surgical patients

Methods: RCTs conducted in adult surgical patients who receive oral nutritional support will be included. The primary outcome will be the incidence of SSIs (within 30 days of surgery or within 90 days for joint replacement surgery). Secondary outcomes will be changes in nutritional status, mortality, health-related quality of life and costs. Literature searches will be conducted in several electronic databases (from inception onwards): MEDLINE, Embase, CINAHL and The Cochrane Central Register of Controlled Trials (CENTRAL). Grey literature will be identified through searching clinical trial registers and dissertation databases. Two reviewers will independently screen all citations, fulltext articles and abstract data. The study methodological quality (or bias) will be appraised using the Cochrane risk of bias tool. If feasible, we will conduct random effects meta-analysis where appropriate.
\end{abstract}

Discussion: This systematic review will evaluate the evidence for pre- and post-surgical intervention with oral nutritional supplements in adults. Findings from this planned review may inform subsequent nutritional interventions for hospitalised patients who undergo surgery.

Systematic review registration: PROSPERO CRD42020140954

Keywords: Surgical site infection, Malnutrition, Nutrition, Surgery

\section{Background}

Surgical site infections (SSIs) are defined by the Centers for Disease Control and Prevention (CDC) as infections that occur after surgery in the part of the body or the incision where the surgery took place. SSIs range from simple wound infections involving the skin and subcutaneous tissues (classified as superficial incisional), to deep soft-tissue infections involving fascia and muscle (classified as deep incisional), or infections of the space or

\footnotetext{
* Correspondence: Nicholas.Ralph@usq.edu.au

${ }^{1}$ School of Nursing and Midwifery, University of Southern Queensland,

Toowoomba, Australia

${ }^{2}$ Division of Research and Innovation, University of Southern Queensland,

Toowoomba, Australia

Full list of author information is available at the end of the article
}

organ manipulated during the surgical procedure (classified as organ/space) [1]. SSIs are among the most common healthcare-associated infections, as the World Health Organisation (WHO) estimates a pooled global SSI incidence rate of $11.2 \%$ [2]. However, variations of SSI incidence are marked. In one North American study of over 750,000 surgical patients, $1 \%$ of procedures resulted in an SSI, with similar incidence rates found in other developed health systems [3, 4]. In a study of 75 , 695 patients in hospitals in the United Kingdom (UK) over a 4-month period, $8 \%$ of patients suffered a healthcare-associated infection, with SSIs making up $15 \%$ of these infections [5]. Depending on whether the surgery is classified as clean, clean/contaminated, 
contaminated or dirty, SSIs can also occur at different rates [6]. Nonetheless, the prevalence of SSIs may be under-estimated as most present within 30 days following a surgical procedure; a proportion of which is likely to develop outside of hospital [7, 8].

SSIs result in delayed wound healing, increased hospital stays, increased use of antibiotics, unnecessary pain and, in extreme cases, death of the patient [9]. SSIs result in delayed wound healing, increased hospital stays, increased use of antibiotics, unnecessary pain, death of the patient in extreme cases, as well as increased health resource use and expenditure depending of the location, depth and severity of the infection $[10,11]$. Factors that contribute to the development of SSI include the patient's health status, the type of surgery conducted and the physical environment where surgical care is provided [12]. Surgical risk factors include emergency surgery or surgeries involving major blood loss, surgery involving contaminated or dirty wounds and prolonged surgery [9]. Patient-level factors include a preoperative American Society of Anesthesiology (ASA) score of III or IV $[13,14]$, high body mass index $(\mathrm{BMI})>35$, malnutrition, advanced age and the pre-existence of immunecompromising conditions or diseases such as diabetes and cancer and malnutrition from inadequate nutritional intake $[15,16]$. Hospitalisation is also associated with a deterioration of nutritional intake in admitted elective surgical patients leading to under-nutrition [17].

Malnutrition can cause poor clinical outcomes from surgery through disruption to physiological and psychological health [18]. Although no universally accepted definition is clear, malnutrition can be broadly described as any imbalance in an individual's nutritional status which affects body composition and/or function: such imbalance might relate to overnutrition through excessive nutritional intake or under-nutrition caused by insufficient nutritional intake or malabsorption [19-21]. Most screening tools typically only distinguish undernourished patients rather than stratifying nutritional status according to whether they are of normal weight or obese/overweight $[22,23]$. Consequently, the stratification of risk among malnourished, normal weight and obese patients is poorly understood. Moreover, compliance with routine screening of nutritional status is inconsistently performed in hospitals, even in countries such as the UK and the United States of America (USA) where it is mandatory on admission [24, 25]. Although the quality of research varies, reported under-nutrition ranges from 30 to $55 \%$ in hospitalised patients in studies conducted across a range of countries [26-30].

Oral nutritional supplements contain either macronutrients (proteins, fats, carbohydrates and amino acids) and/or micronutrients (vitamins and minerals) to supplement the oral diet. Some ingredients in oral nutritional supplements such as proteins, arginine, glutamine, omega-3 fatty acids, vitamins and trace minerals may improve immune function and wound healing [31]. Nutrients such as arginine and glutamine are typically referred to as immunonutrition. Oral nutritional supplements containing macronutrients, micronutrients or a mixture of both are usually prescribed if under-nutrition is diagnosed or the patient is assessed as being nutritionally at-risk, and an improvement in the nutritional status is desired or required [20,32]. To achieve improvements in nutritional status, oral nutritional supplements are usually prescribed for at least 7 days before surgery [33] and up to 4 weeks postoperatively [34]; however, protocols vary. Typically, oral nutritional supplements are taken as liquids up to three times a day with an intake of about $250-600 \mathrm{kcal} /$ daily usually in addition to daily dietary intake [18].

Under-nutrition results from a shortfall between nutrition intake and nutrient requirements leading to tissue loss and changes to normal physiological function [35]. Surgical patients may be undernourished on admission to hospitals [36]. Surgery can exacerbate under-nutrition by causing a systemic inflammatory response which increases metabolic activity, elevates energy consumption, impairs organ function and compromises immunity [37, 38]. Undernourished patients may be at risk of developing SSI $[15,39]$, are at a greater risk of death and morbidity $[40,41]$ and require more hospital resources than normally nourished patients [42, 43]. Oral nutritional supplements containing protein and/or arginine may work to improve nutrition status and reduce the risk of developing an SSI [44-48].

Findings reported from RCTs of oral nutritional supplementation have been mixed across studies of both single and multi-nutrient oral nutritional supplements. For studies investigating the effect of single-nutrient oral nutritional supplementation with a formula of either protein, arginine or amino acids, a reduced rate of postoperative infection complications was reported among head and neck cancer surgery patients but the effect was not statistically significant $[49,50]$. Two further studies were conducted to measure the effect of single-nutrient oral nutritional supplements with no SSIs recorded across the intervention control groups of two studies $[44,45]$. One study reported a statistically insignificant increase in SSIs following single-nutrient oral nutritional supplementation and compared with the control group [51].

Similarly, mixed findings have been reported from RCTs of multi-nutrient oral nutritional supplements defined as a formula of two or more nutrients comprising energy sources and protein, arginine or amino acids. In two studies, fewer SSIs were reported among individuals who received multi-nutrient supplements across two RCTs than those who received routine nutrition [46-48]. 
In similar studies of multi-nutrient oral nutritional supplements, no statistically significant difference in SSI rates was demonstrated [52-54] whilst one study reported that multi-nutrient oral nutritional supplements may increase SSIs relative to routine nutrition [55].

To understand the available evidence and its quality, it is important to search, analyse and report on the role of oral nutritional supplements for preventing SSIs. This systematic review may provide evidence to inform clinical practice, as well as highlighting further areas for investigation. Since SSIs have marked negative effects on the person, health services and society across the globe [56], it is important to identify effective interventions to reduce their incidence. Principally, this review is necessary to help find, appraise and summarise the current evidence on the advantages and disadvantages of oral nutritional supplements and present findings accordingly. Thus, the objective of our planned systematic review is to evaluate oral nutritional supplements on preventing SSIs in adult surgical patients.

\section{Methods/design}

\section{Inclusion and exclusion criteria}

We will include published RCTs that include a statement of peer review including theses, cluster RCTs and $\mathrm{N}$ of 1 trials. We will include unpublished trials located from conference abstracts and proceedings. We will only include studies written in English as we do not have access to scientific translation services. Studies using quasi-randomisation will be excluded. We will include studies of adults 18 or over of any gender who undergo surgery, with or without abnormal body composition, for example, any BMI, of any nutritional status or disease state. We will not exclude patients by surgical type or by hospital setting. However, it is likely that most studies will investigate patients undergoing major surgery such as joint replacement, as oral nutritional supplements are not typically prescribed for individuals undergoing minor surgery such as cataract surgery.

We will include RCTs recruiting people described in the primary report as receiving oral nutritional supplements or no oral nutritional supplements or a placebo in the hospital setting who undergo a surgical procedure. Although we expect most studies will focus on oral nutritional supplements as a treatment for under-nutrition, we will include studies where oral nutritional supplements are prescribed to supplement the diet of normally nourished or obese patients. As the method of assessing and defining nutritional status may vary, we will accept definitions of normal nutrition and malnutrition as used by the study authors.

The primary intervention of interest is oral nutritional supplements containing either macronutrients, micronutrients or a mixture of both. We will exclude studies investigating supplements that do not contain an energy source (carbohydrates, fats, proteins). We will also exclude studies of singlenutrient oral nutritional supplements or parenteral nutrition. We will include RCTs in which the use of oral nutritional supplements during the treatment period, either preoperatively or postoperatively, is the only interventional difference between treatment groups. We anticipate that likely comparisons will include protein-enriched supplements compared with immunonutrition supplements, such as supplements enriched with arginine or glutamine, used during the care pathway and added to standard practice, comparisons of different types/brands of oral nutritional supplements or comparisons of oral nutritional supplements with a placebo or control such as no supplementation. The duration and frequency of oral nutritional supplements compared with a placebo or control will be reported.

We will exclude prebiotics, probiotics and synbiotics as they are ingredients in food that are undigested in the stomach but metabolised in the colon to promote bacterial growth or activity which may benefit health [57]. We will also exclude products which are administered for purposes other than improving nutritional status. As an example, medicinal herbs can be derived from edible foods such as ginger, garlic, dandelion, lavender, fennel, thyme, mint, liquorice, chamomile and St John's Wort and can be taken to achieve a pharmaceutical or pharmacokinetic effect. All medicinal herbs, herbal therapies, homoeopathic substances and zinc supplements will be excluded from the review. We will only review interventions where oral nutritional supplements have been taken to supplement the oral diet as this is a typical approach for targeting improvements in the nutritional status of surgical patients in the hospital setting as well as the phenomena of interest in this review. Patients on total parenteral nutrition (TPN) or receiving supplements via a nasogastric tube will be excluded from the review.

\section{Outcome measures}

We list primary and secondary outcomes below. If a study is otherwise eligible (i.e. correct study design, population and intervention/comparator) but does not report a listed outcome, then we will contact the study authors where possible to establish whether an outcome of interest here was measured but not reported. Studies will be excluded where it is clear that our primary outcome was not measured.

We will report outcome measures at the latest time point available, assumed to be length of follow-up if not specified, and the time point specified in the methods as being of primary interest, if this is different from the latest time point available. For all outcomes, we will class assessment of outcome measures in 2 categories: 
- From up to 30 days after surgery for non-joint replacement surgery

- From up to 90 days after surgery for jointreplacement surgery

The primary outcome of this review is an evaluation of oral nutrition supplements in SSIs. In the latest guidelines for prevention of SSIs [1], the CDC defines SSIs as infections of the incision or organ or space that occur after surgery' (p785) and standardises the types of SSIs into (a) superficial incisional, (b) deep incisional and (c) organ space (CDC 2018b). Despite this, diagnosis of SSIs varies between studies.

We will, therefore, accept the definition used by the original authors to determine:

- The proportion of patients who developed any SSIs before or after discharge from hospital within 30 days following surgery.

- For joint replacement surgery, we will limit reporting to infections which occur within the first 90 days following surgery as per CDC diagnostic guidelines which include this period in the SSI risk period [58].

- We will report any reported incident of SSIs that falls under these definitions as 1 event.

Secondary outcomes are:

- Changes to nutritional status. We will report biological and immunological marker changes compared with intervention and the control groups including total protein $(\mathrm{g} / \mathrm{L})$, albumin $(\mathrm{g} / \mathrm{dl})$, prealbumin $(\mathrm{mg} / \mathrm{dl})$ and total lymphocytes $\left(10^{3}\right.$ in 1 $\mu \mathrm{L} / \mathrm{mm}^{3}$ of blood). We will accept any validated nutritional assessment tool measures taken at least once prior to supplementation and at least once after supplementation is ceased;

- Costs. Any cost effectiveness that relates costs to benefits including but not limited to cost-utility or incremental cost-effectiveness ratio;

- Mortality. We will include death up to 52 weeks following surgery; and

- Health-related quality of life. We will include healthrelated quality of life when reported where a validated scale such as the SF-36 or EQ-5D was used. We will not report ad hoc measures of quality of life that are unlikely to be common to trials and unvalidated.

\section{Search strategy}

We will search the following electronic databases to retrieve reports of relevant randomised clinical trials: the Cochrane Wounds Group Specialised Register (to present), the
Cochrane Central Register of Controlled Trials (CENTRAL) (the Cochrane Library, latest issue), Ovid MEDLINE (1946 to present), Ovid MEDLINE (In-Process \& Other NonIndexed Citations), Ovid Embase (1974 to present) and EBSCO CINAHL Plus (1937 to present).

We will use the provisional search strategy in Additional file 1 to search the Cochrane Central Register of Controlled Trials (CENTRAL). We will combine the Ovid MEDLINE search with the Cochrane Highly Sensitive Search Strategy for identifying randomised trials in MEDLINE: sensitivity- and precision-maximising version (2008 revision) [59]. We will combine the Embase search with the Ovid Embase filter developed by the UK Cochrane Centre [59]. We will combine the CINAHL Plus search with the trial filters developed by the Scottish Intercollegiate Guidelines Network [60]. There will be no restrictions with respect to date of publication or study setting. We will also search the following clinical trial registries: ClinicalTrials.gov, WHO International Clinical Trials Registry Platform and EU Clinical Trials Register.

We will contact the corresponding authors for study information and the manufacturers and distributors of nutritional supplements for product information where this is required. We will try to identify other potentially eligible trials or ancillary publications by searching the reference lists of retrieved included trials as well as relevant systematic reviews, meta-analyses and health technology assessment reports.

\section{Screening}

Two review authors will independently assess the titles and abstracts of the citations retrieved by the searches for relevance. After this initial assessment, we will obtain full text copies of all studies considered to be potentially relevant. In teams of two, review authors will independently check the full papers for eligibility; disagreements will be resolved by discussion and, where required, the input of a third review author. Where required and possible, we will contact study authors where the eligibility of a study is unclear. We will record all reasons for exclusion of studies for which we had obtained full copies. We will complete a PRISMA flowchart to summarise this process and a PRISMA-P checklist is also appended (see Additional file 2) [61]. Where studies have been reported in multiple publications/reports, we will obtain all publications. Whilst the study will be included only once in the review, data will be extracted from all reports to ensure maximal relevant data are obtained.

\section{Extraction}

We will extract and summarise details of the eligible studies using a standardised data extraction sheet. Review authors will extract data independently in pairs and will resolve disagreements by discussion, drawing on a 
third review author where required. Where data are missing from reports, we will attempt to contact the study authors to obtain this information. Where a study with more than two intervention arms is included, only data from intervention and control groups that meet the eligibility criteria will be extracted.

We will extract the following data where possible by treatment group for the pre-specified interventions and outcomes in this review. Outcome data will be collected for relevant time points including:

- Country of origin

- Type of wound and surgery

- Unit of randomisation (per patient)-single wound or multiple wounds on the same patient

- Unit of analysis

- Trial design such as parallel, cluster

- Care setting

- Number of participants randomised to each trial arm

- Eligibility criteria and key baseline participant data

- Details of treatment regimen received by each group

- Commencement, end and duration of treatment

- Details of any co-interventions

- Primary and secondary outcome(s) (with definitions)

- Outcome data for primary and secondary outcomes (by group)

- Duration of follow-up

- Number of withdrawals (by group)

- Blinding (both patient and professional)

- Publication status of study

- Source of funding for trial

- Patient demographics such as gender and age

\section{Quality assessment}

Two review authors will independently assess included studies using the Cochrane tool for assessing risk of bias [62]. This tool addresses six specific domains: sequence generation, allocation concealment, blinding, incomplete data, selective outcome reporting and other issues. In this review we will record issues with unit of analysis, for example, where a cluster trial has been undertaken but analysed at the individual level in the study report. We will assess blinding and completeness of outcome data for each of the review outcomes separately. We note that, since wound healing is a subjective outcome, it can be at high risk of measurement bias when outcome assessment is not blinded. We will present our assessment of risk of bias using two 'risk of bias' summary figures; one which is a summary of bias for each item across all studies, and a second which shows a crosstabulation of each trial by all of the 'risk of bias' items. We will class studies with an assessment of high risk of bias for the randomisation sequence domain and/or the allocation concealment domain and/or the blinded outcome assessment domain (for specified outcome) as being at overall high risk of bias (for specified outcome). Where risk of bias judgement is made based on information from correspondence with trial authors, this will be noted in the risk of bias table. For trials using cluster randomisation, we will also consider the risk of bias in terms of recruitment bias, baseline imbalance, loss of clusters, incorrect analysis and comparability with individually randomised trials [63].

\section{Analysis}

For dichotomous outcomes, we will calculate the odds ratio (OR) with 95\% confidence intervals (CI). For continuously distributed outcome data, we will use the difference in means (MD) with $95 \%$ CIs, if all trials use the same or similar assessment scale. If trials use different assessment scales, we will use the standardised mean difference (SMD) with 95\% CIs. We will only consider mean or median time to healing without survival analysis as a valid outcome if reports specify that all wounds healed, meaning if the trial authors regarded time to healing as a continuous measure as there is no censoring. Time-to-event data such as time-to-complete wound healing will be reported as hazard ratios (HR) where possible in accordance with the methods described in the Cochrane Handbook [64]. If studies reporting time-toevent data such as time to healing do not report a hazard ratio, then, where feasible, we plan to estimate this using other reported outcomes, such as the numbers of events, through the application of available statistical methods [65]. We will use the DerSimonian-Laird method to combine OR as we anticipate using a random effects model due to likely study heterogeneity [65].

Where studies randomise at the participant level and measure outcomes at the wound level, such as wound healing, we will treat the participant as the unit of analysis when the number of wounds assessed appears equal to the number of participants (e.g. one wound per person). There may be instances of clustered data where a proportion of individually randomised trial participants have outcome data collected and reported on multiple wounds. Since not all participants will have multiple wounds, this is not a cluster trial per se but rather a trial that incorrectly includes a mixture of individual and clustered data. Such trials will be noted and the issue will be recorded in the risk of bias assessment. Data will be extracted and presented but will not be the subject of any further analyses.

We will only incorporate clearly conducted fully cluster trials into meta-analyses if the trial has been analysed correctly. Where a cluster trial has been conducted but incorrectly analysed we will record this as part of the 'risk of bias' assessment. If possible, we will approximate 
the correct analyses based on Cochrane Handbook guidance [63] using information on the number of clusters (or groups) randomised to each intervention group or the average (mean) size of each cluster; the outcome data ignoring the cluster design for the total number of individuals (for example, number or proportion of individuals with events, or means and standard deviations) and an estimate of the intracluster (or intraclass) correlation coefficient (ICC). Where multiple trial arms are reported in a single trial, we will include only the relevant arms. If two interventions or more interventions are compared with the control and are eligible for the same meta-analysis, we will pool the intervention arms and compare that with the control. If the study data cannot be analysed correctly, outcome data will be extracted and presented but not analysed further.

It is common to have data missing from trial reports. Excluding participants' post-randomisation from the analysis, or ignoring those participants who are lost to follow up, compromises the randomisation and potentially introduces bias into the trial. Where there are missing data we think should be included in the analyses, we will contact the relevant study authors to request whether these data are available. We will adopt the process detailed by Miller to evaluate the potential bias of bivariate and multivariate comparisons of 'stayers and leavers' [66]. For all secondary outcomes, we will present available data from the study reports/study authors and do not plan to impute missing data. Where measures of variance are missing, we will calculate these wherever possible. If calculation is not possible, we will contact the study authors. Where these measures of variance are not available, we will exclude the study from any relevant meta-analyses that are conducted and discuss the potential implications of its absence from a meta-analysis.

\section{Assessment of heterogeneity}

Assessment of heterogeneity can be a complex, multifaceted process. Firstly, we will consider clinical and methodological heterogeneity, that is, the degree to which the included studies vary in terms of participant, intervention, outcome and characteristics such as length of follow-up. This assessment of clinical and methodological heterogeneity will be supplemented by information regarding statistical heterogeneity, assessed using the chi-squared test where a significance level of $P<$ 0.10 will be considered to indicate statistically significant heterogeneity, in conjunction with the $I^{2}$ measure [67]. $I^{2}$ examines the percentage of total variation across RCTs that is due to heterogeneity rather than chance [67]. In general, $I^{2}$ values of $40 \%$, or less, may not be important [67]; and values of more than $75 \%$, or more, may indicate considerable heterogeneity [64]. We will attempt to explore further where there is evidence of high heterogeneity.

\section{Assessment of bias}

Reporting biases arise when the dissemination of research findings is influenced by the nature and direction of results. Publication bias is one of a number of possible causes of small study effects, that is, a tendency for estimates of the intervention effect to be more beneficial in smaller RCTs. We will produce funnel plots as a visual assessment of whether small study effects may be present in a meta-analysis and as a means of estimating the intervention effects from individual RCT against some measure of each trial's size or precision [68]. We plan to present funnel plots for meta-analyses comprising 10 RCTs or more.

\section{Outcomes}

Details of included studies will be combined in a narrative review according to type of comparator, possibly by location/type of wound and then by outcomes by time period. Clinical and methodological heterogeneity will be considered and pooling undertaken when studies appear appropriately similar in terms of wound type, intervention type, duration of follow-up and outcome type.

We are unable to pre-specify the amount of clinical, methodological and statistical heterogeneity in the included studies but it might be extensive. Thus, we anticipate using a random effects approach for meta-analysis. Conducting meta-analysis with a fixed effect model in the presence of even minor heterogeneity may provide overly narrow confidence intervals. We will only use a fixed effect approach when clinical and methodological heterogeneity are assessed to be minimal, and the assumption that a single underlying treatment effect is being estimated holds. Chi-squared and $I^{2}$ will be used to quantify heterogeneity but will not be used to guide choice of model for meta-analysis. We will exercise caution when meta-analysed data are at risk of small study effects because a random effects model may be unsuitable. In this case, or where there are other reasons to question the selection of a fixed effect or random effects model, we will assess the impact of the approach using sensitivity analyses to compare results from alternate models. We will report any evidence that suggests that the use of a particular model might not be robust. We may meta-analyse except when there is thought to be extensive heterogeneity. We will attempt to explore the causes behind this using meta-regression, if possible [69].

Data will be presented using forest plots where possible. For dichotomous outcomes, we will present the summary estimate as an odds ratio (OR) with 95\% CI. 
Where continuous outcomes are measured in the same way across studies, we plan to present a pooled MD with 95\% CI; we plan to pool [70] SMD estimates where studies measure the same outcome using different methods. For time-to-event data, we plan to plot and, if appropriate, to pool, estimates of hazard ratios and $95 \%$ CIs as presented in the study reports using the generic inverse variance method. Where time to healing is analysed as a continuous measure but it is not clear if all wounds healed, use of the outcome in the study will be documented but data will not be summarised or used in any meta-analysis.

We will present the main results of the review in 'summary of findings' tables; one table per comparison. The tables will contain key information concerning the quality of the evidence, the magnitude of the effects of the interventions examined and the sum of the available data for the main outcomes [71]. The 'summary of findings' tables will also include an overall grading of the evidence related to each of the main outcomes using the GRADE (Grades of Recommendation, Assessment, Development and Evaluation) approach [72]. The GRADE approach defines the quality of a body of evidence as the extent to which one can be confident that an estimate of effect or association is close to the true effect. The quality of a body of evidence involves consideration of within-trial risk of bias (methodological quality), inconsistency between studies (heterogeneity), directness of evidence (generalisability of population or outcomes), precision of effect estimates and risk of publication bias [70]. We will downgrade evidence using an approach adapted from Dijkers (see Table 1) [73].

We plan to present the following outcomes in the 'summary of findings' tables: SSIs incidence, changes to nutritional status, cost, mortality and health-related quality of life. If sufficient data are available, we will carry out subgroup analyses in order to determine potentially important differences in the characteristics of interventions or the effect of interventions on different groups. We will assess potential heterogeneity. We will consider the following subgroups:

Table 1 Factors that may lead to downgrading or upgrading of evidence in the GRADE approach

\begin{tabular}{|c|c|}
\hline $\begin{array}{l}\text { Criteria for } \\
\text { downgrading }\end{array}$ & By how many levels \\
\hline 1. Serious risk of bias & $\begin{array}{l}\text { Serious }(-1) \text { or very serious }(-2) \text { limitation } \\
\text { to study quality }\end{array}$ \\
\hline $\begin{array}{l}\text { 2. Serious inconsistence } \\
\text { between studies }\end{array}$ & $\begin{array}{l}\text { Important inconsistency, lack of agreement } \\
\text { between studies or conflicting results }(-1)\end{array}$ \\
\hline 3. Serious indirectness & $\begin{array}{l}\text { Uncertainty with generalisability of population } \\
\text { or outcomes }(-1) \text { or with both population and } \\
\text { outcomes }(-2)\end{array}$ \\
\hline 4. Serious imprecision & Imprecise or sparse data $(-1)$ \\
\hline 5. Likely publication bias & High probability of reporting bias $(-1)$ \\
\hline
\end{tabular}

1. Age such as $<18$ years, $\geq 18$ years and $<65$ years and $\geq 65$ years

2. Gender

3. Type of surgery such as clean, clean/contaminated, contaminated or dirty

4. Body composition according to CDC [74] definitions of underweight, normal weight and overweight such as BMI $>30$ obese

5. Type of intervention such as protein-based or arginine-based or both [75]

6. Degree of nutrition status such as classifications of under-nutrition over nutrition or normal nutrition as assessed by any nutrition assessment tool or as defined by study authors [19-21]

7. Treatment regimen of nutrition supplement such as time of initiation and period of dosing [76]

8. Low risk of bias versus unclear or high risk of bias

9. Biomarker levels (normal versus abnormal ranges)

\section{Sensitivity analyses}

Where possible, we plan to perform sensitivity analyses to explore the impact or influence of key assumptions or variations on effect estimates related to different nutritional assessment tools used to define nutrition status.

\section{Discussion}

Since evaluating nutritional status, complications and interventions are complex; we anticipate there may be few studies that appropriately address the research question. Therefore, we will conduct preliminary searches to scope the literature and systematically refine the search to ensure inclusion of all relevant literature. Additionally, searching and retrieving theses may prove to be complex. However, we will use ProQuest Theses and Dissertation database as well as the Gray Matters Application to minimise the chance of missing potential studies. Given heterogeneity between interventions, outcomes and research designs is likely, it may not be appropriate to meta-analyse the studies. Also, without large, cluster randomised controlled trials distinguishing interventional effectiveness across surgical demographics will be challenging. We will perform an analysis of evidence quality to counter this potential gap.

\section{Supplementary information}

Supplementary information accompanies this paper at https://doi.org/10. 1186/s13643-020-01293-x

Additional file 1. Provisional search strategy

Additional file 2. PRISMA-P 2015 Checklist

\footnotetext{
Abbreviations

ASA: American Society of Anesthesiology; BMI: Body mass index;

CDC: Centers for Disease Control and Prevention; Cl: Confidence intervals;

GRADE: Grades of Recommendation, Assessment, Development and

Evaluation; HR: Hazard ratios; ICC: Intracluster (or intraclass) correlation
} 
coefficient; MD: Difference in the means; OR: Odds ratio; RCT: Randomised controlled trial; SMD: Standardised mean difference; SSI: Surgical site infection; TPN: Total parenteral nutrition; UK: United Kingdom; USA: United States of America; WHO: World Health Organisation

\section{Acknowledgements}

We are thankful to Dave Porter for his assistance in formatting the publication for presentation.

\section{Authors' contributions}

NR conceived the review question, developed the protocol, coordinated the protocol development, produced the first draft of the protocol, contributed to writing or editing the protocol, advised on the protocol, approved the final version of the protocol prior to submission and is a guarantor of the protocol. LB developed the protocol, coordinated the protocol development, produced the first draft of the protocol, contributed to writing or editing the protocol, advised on the protocol and approved the final version of the protocol prior to submission. KMK conceived the review question, developed the protocol, coordinated the protocol development, produced the first draft of the protocol, contributed to writing or editing the protocol and approved the final version of the protocol prior to submission. JD developed the protocol, produced the first draft of the protocol, contributed to writing or editing the protocol, advised on the protocol and approved the final version of the protocol prior to submission. SO developed the protocol, produced the first draft of the protocol, contributed to writing or editing the protocol, advised on the protocol and approved the final version of the protocol prior to submission. VT developed the protocol, contributed to writing or editing the protocol, advised on the protocol and approved the final version of the protocol prior to submission. KLE developed the protocol, contributed to writing or editing the protocol, advised on the protocol and approved the final version of the protocol prior to submission. RK developed the protocol, contributed to writing or editing the protocol, advised on the protocol and approved the final version of the protocol prior to submission. EB contributed to writing or editing the protocol, advised on the protocol and approved the final version of the protocol prior to submission. All authors read and approved the final manuscript.

\section{Authors' information}

Nicholas Ralph, Kristy McKillop, Sonya Osborne, Jed Duff, Victoria Terry and Karen-Leigh Edward are all researchers and registered nurses with experience in perioperative and surgical care. Lindsay Brown is a Professor of Biomedical Science and has an international research profile in functional foods and nutrition. Edward Barui is a Consultant Orthopaedic Surgeon. Rachel King is a statistician.

\section{Funding}

Kristy McKillop's doctoral studies were supported by a scholarship administered through the University of Southern Queensland and funded by the Federal Government of Australia.

\section{Availability of data and materials \\ Not applicable}

\section{Ethics approval and consent to participate}

Not applicable

\section{Consent for publication}

Not applicable

\section{Competing interests}

The authors declare that they have no competing interests.

\section{Author details}

'School of Nursing and Midwifery, University of Southern Queensland, Toowoomba, Australia. ${ }^{2}$ Division of Research and Innovation, University of Southern Queensland, Toowoomba, Australia. ${ }^{3}$ Faculty of Health, University of Technology Sydney, Ultimo, Australia. ${ }^{4}$ School of Health and Wellbeing, University of Southern Queensland, Toowoomba, Australia. ${ }^{5}$ St Vincent's Private Hospital, Toowoomba, Australia. ${ }^{6}$ School of Nursing and Midwifery, The University of Newcastle, Callaghan, Australia. 'School of Public Health and Social Work, Institute of Health and Biomedical Innovation, Queensland University of Technology, Brisbane, Australia. ${ }^{8}$ School of Nursing, Swinburne
University, Melbourne, Australia. ${ }^{9}$ School of Sciences, University of Southern Queensland, Toowoomba, Australia.

Received: 2 July 2019 Accepted: 9 February 2020

Published online: 20 February 2020

\section{References}

1. Berrios-Torres SI, Umscheid CA, Bratzler DW, Leas B, Stone EC, Kelz RR, et al, Centers for disease control and prevention guideline for the prevention of surgical site infection, 2017. JAMA Surgery. 2017;152(8):784-91.

2. Leaper DJ, Edmiston CE. World Health Organization: global guidelines for the prevention of surgical site infection. Journal of Hospital Infection 2017. p. 135-6.

3. Astagneau P, L'Hériteau F, Daniel F, Parneix P, Venier AG, Malavaud S, et al. Reducing surgical site infection incidence through a network: results from the French ISO-RAISIN surveillance system. J Hosp Infect. 2009;72(2):127-34.

4. De Lissovoy G, Fraeman K, Hutchins V, Murphy D, Song D, Vaughn BB. Surgical site infection: incidence and impact on hospital utilization and treatment costs. Am J Infect Control. 2009;37(5):387-97.

5. Smyth ET, Mcllvenny G, Enstone JE, Emmerson AM, Humphreys $\mathrm{H}_{\text {, }}$ Fitzpatrick F, et al. Four country healthcare associated infection prevalence survey 2006: overview of the results. J Hosp Infect. 2008;69(3):230-48.

6. Ortega G, Rhee DS, Papandria DJ, Yang J, Ibrahim AM, Shore AD, et al. An evaluation of surgical site infections by wound classification system using the ACS-NSQIP. J Surg Res. 2012;174(1):33-8

7. Gibbons C, Bruce J, Carpenter J, Wilson AP, Wilson J, Pearson A, et al. Identification of risk factors by systematic review and development of riskadjusted models for surgical site infection. Health Technol Assess. 2011; 15(30):1-156 iii-iv.

8. Bruce D, Laurance I, McGuiness M, Ridley M, Goldswain P. Nutritional supplements after hip fracture: poor compliance limits effectiveness. Clinical nutrition (Edinburgh, Scotland). 2003;22(5):497-500.

9. Reichman DE, Greenberg JA. Reducing surgical site infections: a review. Reviews in Obstetrics and Gynecology 2009. p. 212-21.

10. Fry DE. The economic costs of surgical site infection. Surg Infect. 2002; 3(Suppl 1):S37-43.

11. Bratzler DW, Houck PM. Antimicrobial prophylaxis for surgery: an advisory statement from the National Surgical Infection Prevention Project. Clin Infect Dis. 2004;38(12):1706-15.

12. Barie PS. Surgical site infections: epidemiology and prevention. Surg Infect. 2002;3(Suppl 1):S9-21.

13. Asensio A, Ramos A, Cuervas-Mons V, Cordero E, Sanchez-Turrion V, Blanes $M$, et al. Effect of antibiotic prophylaxis on the risk of surgical site infection in orthotopic liver transplant. Liver Transpl. 2008;14(6):799-805.

14. Dorschner $P$, McElroy LM, Ison MG. Nosocomial infections within the first month of solid organ transplantation. Transpl Infect Dis. 2014;16(2):171-87.

15. Blumetti J, Luu M, Sarosi G, Hartless K, McFarlin J, Parker B, et al. Surgical site infections after colorectal surgery: do risk factors vary depending on the type of infection considered? Surgery. 2007;142(5):704-11.

16. Watanabe A, Kohnoe S, Shimabukuro R, Yamanaka T, Iso Y, Baba H, et al. Risk factors associated with surgical site infection in upper and lower gastrointestinal surgery. Surg Today. 2008;38(5):404-12.

17. Manning F, Harris K, Duncan R, Walton K, Bracks J, Larby L, et al. Additional feeding assistance improves the energy and protein intakes of hospitalised elderly patients. A health services evaluation. Appetite. 2012:59(2):471-7.

18. Stratton RJ, Elia M. Encouraging appropriate, evidence-based use of oral nutritional supplements. Proc Nutr Soc. 2010;69(4):477-87.

19. Barker LA, Gout BS, Crowe TC. Hospital malnutrition: prevalence, identification and impact on patients and the healthcare system. Int J Environ Res Public Health. 2011;8(2):514-27.

20. Cederholm T, Bosaeus I, Barazzoni R, Bauer J, Van Gossum A, Klek S, et al. Diagnostic criteria for malnutrition - an ESPEN consensus statement. Clin Nutr. 2015:34(3):335-40.

21. White JV, Guenter P, Jensen G, Malone A, Schofield M. Consensus statement of the Academy of Nutrition and Dietetics/American Society for Parenteral and Enteral Nutrition: characteristics recommended for the identification and documentation of adult malnutrition (undernutrition). J Acad Nutr Diet. 2012:112(5):730-8.

22. Kondrup J, Rasmussen HH, Hamberg O, Stanga Z. Nutritional risk screening (NRS 2002): a new method based on an analysis of controlled clinical trials. Clin Nutr. 2003;22(3):321-36. 
23. Stratton RJ, Hackston A, Longmore D, Dixon R, Price S, Stroud M, et al. Malnutrition in hospital outpatients and inpatients: prevalence, concurrent validity and ease of use of the 'malnutrition universal screening tool' ('MUST') for adults. Br J Nutr. 2004:92(5):799-808.

24. Elia M, Zellipour L, Stratton RJ. To screen or not to screen for adult malnutrition? Clin Nutr. 2005;24(6):867-84.

25. Patel V, Romano M, Corkins M, DiMaria-Ghalili R, Earthman C, Malone A, et al. Nutrition screening and assessment in hospitalized patients: a survey of current practice in the United States. Nutr Clin Pract. 2014;29(4):483-90.

26. Correia MI, Waitzberg DL. The impact of malnutrition on morbidity, mortality, length of hospital stay and costs evaluated through a multivariate model analysis. Clinical nutrition (Edinburgh, Scotland) 2003:22(3):235-9.

27. Correia Misabel TD, Campos Antonio Carlos L. Prevalence of hospital malnutrition in Latin America:: the multicenter ELAN study. Nutrition. 2003; 19(10):823-5.

28. Edington J, Boorman J, Durrant ER, Perkins A, Giffin CV, James R, et al. Prevalence of malnutrition on admission to four hospitals in England. The Malnutrition Prevalence Group. Clin Nutr. 2000;19(3):191-5.

29. Middleton MH, Nazarenko G, Nivison-Smith I, Smerdely P. Prevalence of malnutrition and 12-month incidence of mortality in two Sydney teaching hospitals. Intern Med J. 2001;31(8):455-61.

30. Ray S, Laur C, Golubic R. Malnutrition in healthcare institutions: a review of the prevalence of under-nutrition in hospitals and care homes since 1994 in England. Clin Nutr. 2014;33(5):829-35.

31. Chow O, Barbul A. Immunonutrition: role in wound healing and tissue regeneration. Advances in Wound Care 2014. p. 46-53.

32. National Institute for $\mathrm{H}$, Care Excellence. Nutrition support for adults: oral nutrition support, enteral tube feeding and parenteral nutrition. niceorguk/ guidance/cg32 2006.

33. Botella-Carretero JI, Iglesias B, Balsa JA, Arrieta F, Zamarron I, Vazquez C. Perioperative oral nutritional supplements in normally or mildly undernourished geriatric patients submitted to surgery for hip fracture: a randomized clinical trial. Clin Nutr. 2010;29(5):574-9.

34. Myint MW, Wu J, Wong E, Chan SP, To TS, Chau MW, et al. Clinical benefits of oral nutritional supplementation for elderly hip fracture patients: a single blind randomised controlled trial. Age Ageing. 2013;42(1):39-45.

35. De Bandt JP. Understanding the pathophysiology of malnutrition for better treatment. Ann Pharm Fr. 2015;73(5):332-5.

36. Kahokehr Arman A, Tarik S, Kit W, Vahe S, Plank Lindsay D, Hill Andrew G. Prevalence of malnutrition on admission to hospital - Acute and elective general surgical patients. European e-Journal of Clinical Nutrition and Metabolism. 2010;5(1):e21-e5.

37. Mullen BM, Mattox DE, Von Hoff DD, Hearne EM. The effect of preoperative adriamycin and dihydroxyanthracenedione on wound healing. Laryngoscope. 1981;91(9 Pt 1):1436-43.

38. Cerra FB. Hypermetabolism, organ failure, and metabolic support. Surgery. 1987;101(1):1-14.

39. Gibbs J, Cull W, Henderson W, Daley J, Hur K, Khuri SF. Preoperative serum albumin level as a predictor of operative mortality and morbidity: results from the National VA Surgical Risk Study. Arch Surg. 1999;134(1):36-42.

40. Inagaki E, Farber A, Eslami MH, Kalish J, Rybin DV, Doros G, et al. Preoperative hypoalbuminemia is associated with poor clinical outcomes after open and endovascular abdominal aortic aneurysm repair. J Vasc Surg. 2016;63(6 (Suppl)):67-8S.

41. Van Wissen J, Van Stijn MF, Doodeman HJ, Houdijk AP. Mini nutritional assessment and mortality after hip fracture surgery in the elderly. J Nutr Health Aging. 2016;20(9):964-8

42. Amaral TF, Matos LC, Tavares MM, Subtil A, Martins R, Nazare M, et al. The economic impact of disease-related malnutrition at hospital admission. Clin Nutr. 2007;26(6):778-84.

43. Garcia GH, Fu MC, Dines DM, Craig EV, Gulotta LV. Malnutrition: a marker for increased complications, mortality, and length of stay after total shoulder arthroplasty. J Shoulder Elb Surg. 2016;25(2):193-200.

44. de Luis DA, Izaola O, Cuellar L, Terroba MC, Aller R. Randomized clinical trial with an enteral arginine-enhanced formula in early postsurgical head and neck cancer patients. Eur J Clin Nutr. 2004;58(11):1505-8.

45. De Luis DA, Izaola O, Cuellar L, Terroba MC, Martin T, Aller R. High dose of arginine enhanced enteral nutrition in postsurgical head and neck cancer patients. A randomized clinical trial. Eur Rev Med Pharmacol Sci. 2009;13(4): 279-83.
46. Celik JB, Gezginc K, Ozcelik K, Celik C. The role of immunonutrition in gynecologic oncologic surgery. Eur J Gynaecol Oncol. 2009;30(4):418-21.

47. Klek S, Sierzega M, Szybinski P, Szczepanek K, Scislo L, Walewska E, et al. The immunomodulating enteral nutrition in malnourished surgical patients - a prospective, randomized, double-blind clinical trial. Clinical nutrition (Edinburgh, Scotland). 2011;30(3):282-8.

48. Snyderman CH, Kachman K, Molseed L, Wagner R, D'Amico F, Bumpous J, et al. Reduced postoperative infections with an immune-enhancing nutritional supplement. Laryngoscope. 1999;109(6):915-21.

49. Casas-Rodera P, Gomez-Candela C, Benitez S, Mateo R, Armero M, Castillo R, et al. Immunoenhanced enteral nutrition formulas in head and neck cancer surgery: a prospective, randomized clinical trial. Nutr Hosp. 2008;23(2):105-10

50. de Luis DA, Aller R, Izaola O, Cuellar L, Terroba MC. Postsurgery enteral nutrition in head and neck cancer patients. Eur J Clin Nutr. 2002;56(11): 1126-9.

51. Takeuchi H, Ikeuchi S, Kawaguchi Y, Kitagawa Y, Isobe $Y$, Kubochi K, et al. Clinical significance of perioperative immunonutrition for patients with esophageal cancer. World J Surg. 2007;31(11):2160-7.

52. Falewee MN, Schilf A, Boufflers E, Cartier C, Bachmann P, Pressoir M, et al, Reduced infections with perioperative immunonutrition in head and neck cancer: exploratory results of a multicenter, prospective, randomized, doubleblind study. Clinical nutrition (Edinburgh, Scotland). 2014;33(5):776-84.

53. Gianotti L, Braga M, Nespoli L, Radaelli G, Beneduce A, Di Carlo V. A randomized controlled trial of preoperative oral supplementation with a specialized diet in patients with gastrointestinal cancer. Gastroenterology. 2002;122(7):1763-70.

54. Tepaske R, Velthuis $H$, Oudemans-Van Straaten HM, Heisterkamp SH, Van Deventer SJ, Ince C, et al. Effect of preoperative oral immuneenhancing nutritional supplement on patients at high risk of infection after cardiac surgery: a randomised placebo-controlled trial. Lancet. 2001;358(9283):696-701.

55. Fujitani K, Tsujinaka T, Fujita J, Miyashiro I, Imamura H, Kimura Y, et al. Prospective randomized trial of preoperative enteral immunonutrition followed by elective total gastrectomy for gastric cancer. Br J Surg. 2012; 99(5):621-9.

56. Worth LJ, Bull AL, Spelman T, Brett J, Richards MJ. Diminishing surgical site infections in Australia: time trends in infection rates, pathogens and antimicrobial resistance using a comprehensive Victorian surveillance program, 2002-2013. Infect Control Hosp Epidemiol. 2015;36(4):409-16.

57. Ford AC, Quigley EM, Lacy BE, Lembo AJ, Saito YA, Schiller LR, et al. Efficacy of prebiotics, probiotics, and synbiotics in irritable bowel syndrome and chronic idiopathic constipation: systematic review and meta-analysis. Am J Gastroenterol. 2014;109(10):1547-61 quiz 6, 62.

58. Horan TC, Gaynes RP, Martone WJ, Jarvis WR, Emori TG. CDC definitions of nosocomial surgical site infections, 1992: a modification of CDC definitions of surgical wound infections. Infect Control Hosp Epidemiol. 1992;13(10): 606-8.

59. Lefebvre C, Manheimer E, Glanville J. Chapter 6: Searching for studies. In: Higgins JP, Green S, editor(s). Cochrane Handbook for Systematic Reviews of Interventions Version 5.1.0 (updated March 2011). The Cochrane Collaboration, 2011. Available from handbookcochraneorg.

60. Scottish Intercollegiate Guidelines N. Search filters. www.sign.ac.uk/searchfiltershtml 2017

61. Liberati A, Altman DG, Tetzlaff J, Mulrow C, Gotzsche PC, loannidis JP, et al. The PRISMA statement for reporting systematic reviews and meta-analyses of studies that evaluate health care interventions: explanation and elaboration. PLoS Med. 2009;6:e1000100-e.

62. Higgins JP, Altman DG, Sterne JA, editor. Chapter 8: assessing risk of bias in included studies. In: Higgins JP, Green S, editor(s). Cochrane Handbook for Systematic Reviews of Interventions Version 5.1.0 (updated March 2011). The Cochrane Collaboration, 2011. Available from handbookcochraneorg.

63. Higgins JP, Altman DG, Sterne JA, editor. Chapter 16: Special topics in statistics. In: Higgins JP, Green S, editor(s). Cochrane Handbook for Systematic Reviews of Interventions Version 5.1.0 (updated March 2011). The Cochrane Collaboration, 2011. Available from www.cochranehandbookorg.

64. Deeks JJ, Higgins JP, Altman DG, editor. Chapter 9: Analysing data and undertaking meta-analyses. In: Higgins JP, Green S, editor(s). Cochrane Handbook for Systematic Reviews of Interventions Version 5.1.0 (updated March 2011). The Cochrane Collaboration, 2011. Available from handbookcochraneorg. 
65. Parmar MK, Torri V, Stewart L. Extracting summary statistics to perform meta-analysis of the published literature for survival endpoints. Stat Med. 1998;17:2815-34.

66. Miller RB, Wright DW. Detecting and correcting attrition bias in longitudinal family research. J Marriage Fam. 1995;57(4):921-9.

67. Higgins JP, Thompson SG, Deeks JJ, Altman DG. Measuring inconsistency in meta-analyses. BMJ. 2003;327(7414):557-60.

68. Sterne JA, Sutton AJ, loannidis JP, Terrin N, Jones DR, Lau J, et al. Recommendations for examining and interpreting funnel plot asymmetry in meta-analyses of randomised controlled trials. BMJ. 2011;343:d4002-d.

69. Thompson SG, Sharp SJ. Explaining heterogeneity in meta-analysis: a comparison of methods. Stat Med. 1999;18(20):2693-708.

70. Schünemann HJ, Oxman AD, Higgins JP, Deeks JJ, Glasziou P, Guyatt GH. Chapter 12: interpreting results and drawing conclusions. In: Higgins JP, Green S, editor(s). Cochrane Handbook for Systematic Reviews of Interventions Version 5.1.0 (updated March 2011). The Cochrane Collaboration, 2011. Available from handbookcochraneorg.

71. Schünemann HJ, Oxman AD, Higgins JP, Vist GE, Glasziou P, Guyatt GH. Chapter 11: presenting results and 'summary of findings' tables. In: Higgins JP, Green S, editor(s). Cochrane Handbook for Systematic Reviews of Interventions Version 5.1.0 (updated March 2011). The Cochrane Collaboration, 2011. Available from handbookcochraneorg.

72. Guyatt G, Oxman AD, Akl EA, Kunz R, Vist G, Brozek J, et al. GRADE guidelines 1: introduction - GRADE evidence profiles and summary of findings tables. J Clin Epidemiol. 2011;64(4):383-94.

73. Dijkers M. Introducing GRADE: a systematic approach to rating evidence in systematic reviews and to guideline development. KT Update. 2013;1(5):1-9.

74. Centers for Disease Control and Prevention. Defining Adult Overweight and Obesity. https://www.cdc.gov/obesity/adult/defininghtml 2018.

75. Hegazi RA, Hustead DS, Evans DC. Preoperative standard oral nutrition supplements vs immunonutrition: results of a systematic review and metaanalysis. J Am Coll Surg. 2014;219(5):1078-87.

76. Font-Vizcarra L, Lozano L, Rios J, Forga MT, Soriano A. Preoperative nutritional status and post-operative infection in total knee replacements: a prospective study of 213 patients. Int J Artif Organs. 2011;34(9):876-81.

\section{Publisher's Note}

Springer Nature remains neutral with regard to jurisdictional claims in published maps and institutional affiliations.

Ready to submit your research? Choose BMC and benefit from:

- fast, convenient online submission

- thorough peer review by experienced researchers in your field

- rapid publication on acceptance

- support for research data, including large and complex data types

- gold Open Access which fosters wider collaboration and increased citations

- maximum visibility for your research: over $100 \mathrm{M}$ website views per year

At $\mathrm{BMC}$, research is always in progress.

Learn more biomedcentral.com/submissions 